\title{
Ever-increasing resolution
}

\section{Overcoming the limitations of spatial and temporal resolution to image within a cell is no easy feat. Kelly Rae Chi examines the latest diffraction-busting technologies.}

For many years it was a source of frustration for biologists that the internal components of a cell were practically invisible to them. Researchers believed that the wavelength of light determined a fundamental limit to the resolution of optical microscopes.

However, it now seems that the wavelength of light was not such a limiting factor after all. Super-resolution technology allows researchers to see details that are difficult or impossible to image with conventional light microscopes - at resolutions of 100 nanometres or better.

“There's a huge explosion of interest and progress," says W. E. Moerner, a professor of chemistry and applied physics at Stanford University in California. "That makes it very exciting to watch and to participate in."

Although the theory behind super-resolution is advancing, and many different techniques are now validated, commercialization of these new microscopes is to some extent in its infancy so much so that many research teams still tackle super-resolution using their own lenses, lasers and algorithms.

\section{Upping commercialization}

The past year or so has been particularly active in terms of commercialization. Throughout 2008, Applied Precision of Issaquah, Washington, tested prototypes of its DeltaVision OMX system. The final version is available this year and is already installed in 11 laboratories. It uses three-dimensional structured-illumination microscopy, or 3D-SIM, which illuminates a sample with a series of light patterns that look like bar codes. The low-resolution light patterns reflect off the fine structure of the sample to create moiré fringes. By applying bar codes in different orientations and processing the reflections using computer algorithms, the microscope generates a high-resolution image of the underlying structure.

Carl Zeiss, headquartered in Oberkochen, Germany, is also using the SIM method in its system, ELYRA S.1, to be launched in January. And the company is launching ELYRA P.1, which is based on photoactivated localization microscopy (PALM). This uses photoactivatable proteins that are scattered throughout the sample. Taking multiple frames of the photoactivated dots, and combining them into a high-resolution image, the ELYRA P.1 produces a resolution of around $20 \mathrm{~nm}$. Zeiss also offers a single system, the ELYRA PS.1, that combines laser scanning microscopy, PALM and SIM.

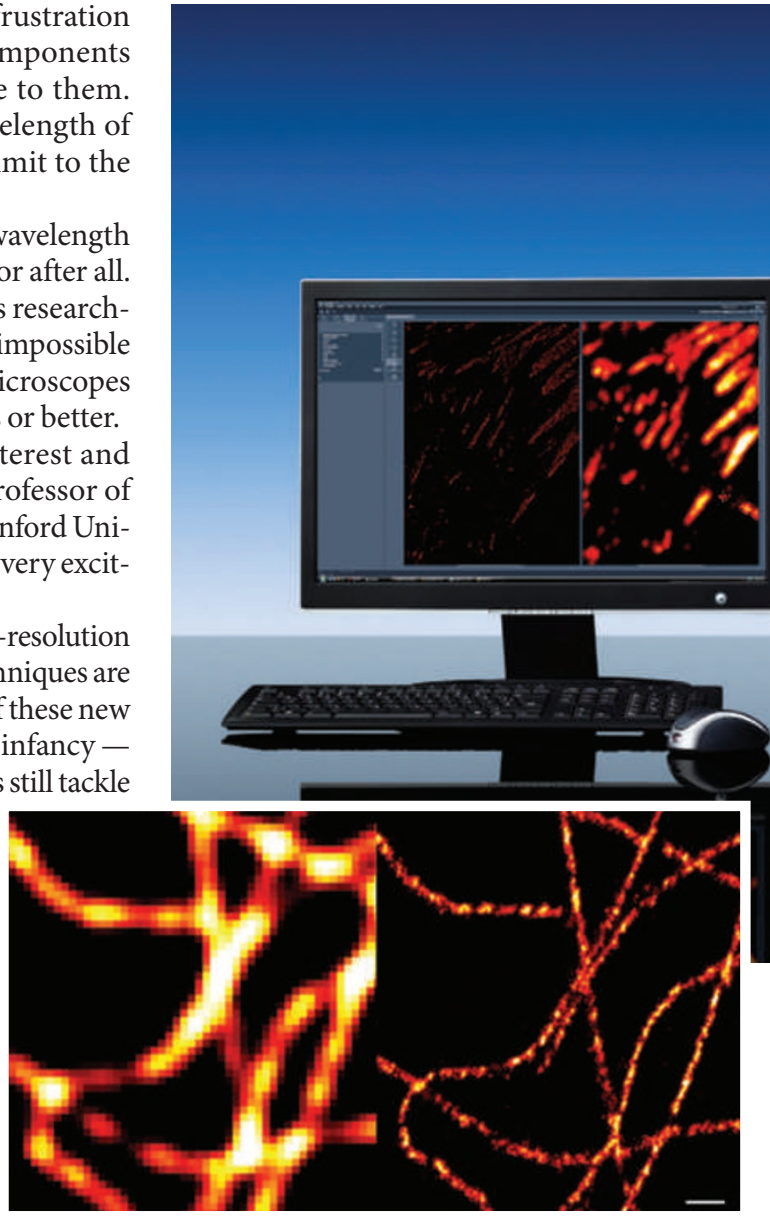

Leica Microsystems in Wetzlar, Germany, is using a variation of stimulated emission depletion (STED) microscopy in its newest super-resolution system. The technique uses a laser beam to excite fluorescent dyes, or fluorophores, within the sample. As with a normal laser scanning microscope, the size of the excited spot determines the resolution of the microscope. In STED microscopy, to improve resolution and narrow the focus of the beam, an excitatory laser pulse is immediately followed by a ring-shaped depletion laser pulse to leave molecules in a smaller region in the centre of the ring in an excited state. This allows resolution of structural details in the $100 \mathrm{~nm}$ range. In Leica's new system, TCS STED CW, the excitatory and depletion laser beams are continuous and overlayed rather than pulsed. Whereas the TCS STED microscope was priced at around US\$1 million, the cost of the TCS STED CW microscope will be closer to the price range of high-end confocal microscopes.

The lasers in TCS STED CW microscopy use the intermediate range of the electromagnetic

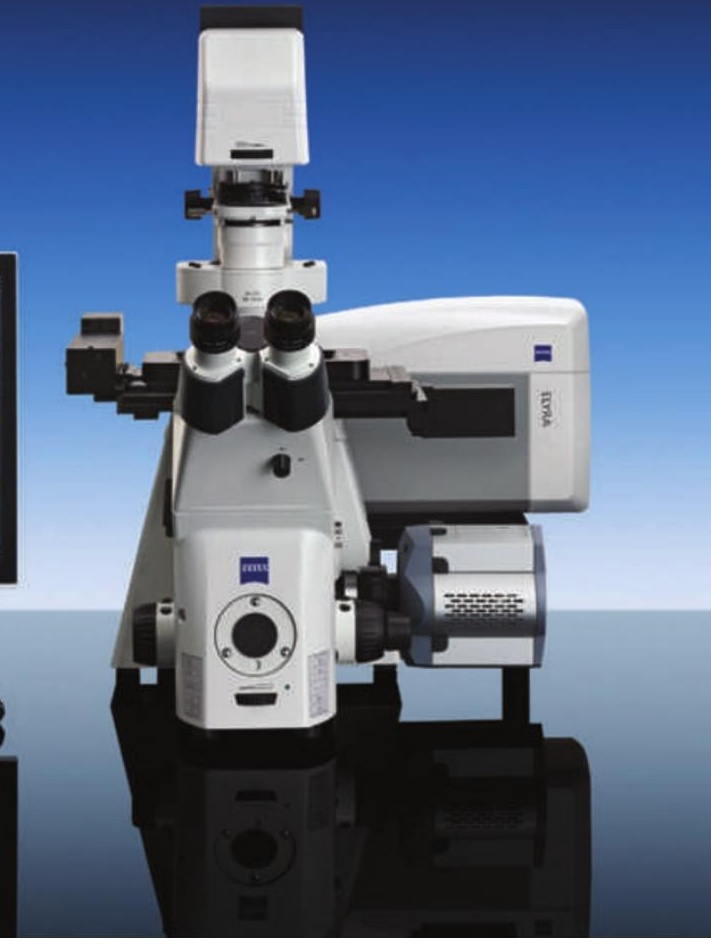

Zeiss's ELYRA system is one of several superresolution microscopes taking microscopy to new levels (near right). Scale bar corresponds to $1 \mathrm{~mm}$.

spectrum, which means the system works with common fluorescents such as enhanced yellow fluorescent protein (EYFP), Alexa Fluor 488 and fluorescein isothiocyanate (FITC). "As long as it's a good confocal sample, it's a good STED sample as well," says Tanjef Szellas, product manager for super-resolution at Leica in Mannheim in Germany.

"Building or buying a confocal without a STED option doesn't make much sense," says Stefan Hell, a physicist at the Max Planck Institute for Biophysical Chemistry in Göttingen, Germany, who pioneered the super-resolution movement by creating STED microscopy and other forms of imaging that bypass the limits of diffraction - about $200 \mathrm{~nm}$ (see 'Breaking the light barrier', overleaf). "The commercial hurdles, in terms of costs and system complexity for basic STED microscopy, are gone."

Nikon, headquartered in Tokyo, is using a super-resolution approach called stochastic optical reconstruction microscopy (STORM) - a similar system to PALM - developed in the lab of Xiaowei Zhuang, a physicist at Harvard University in Cambridge, Massachusetts. STORM uses photoswitchable fluorescent dyes that are activated and deactivated 
by different coloured light. By generating overlapping images in different colours and compiling them, the system can produce threedimensional images that have a spatial resolution ten times better than confocal images in each dimension, Zhuang says. Nikon's instrument, called N-STORM, should be available by May 2010.

\section{Technology tweaks}

The pioneers of super-resolution microscopy are continuing to improve their methods with better sample preparation, a few strategically placed pieces of hardware and more sophisticated algorithms.

One way to achieve greater resolution in fixed samples is to cut thinner slices of tissue. Some groups, including Zhuang's, are collaborating to combine STORM with array tomography, which involves embedding fixed tissue in resin and cutting ultrathin (50-200 nm) slices. "Array tomography is a potential adjunct to all the super-resolution methods," says Stephen Smith of Stanford University in Palo Alto, California, who first described the method. Using this combination, his group has achieved a 14 -fold improvement in axial resolution, and twofold improvements in the each of the two dimensions, compared with confocal imaging.

Other combination strategies are possible. For example, two-photon excitation microscopy, an established method that allows researchers to see further into tissues, can be combined with STED microscopy. Earlier this year, Hell's group used the technique on

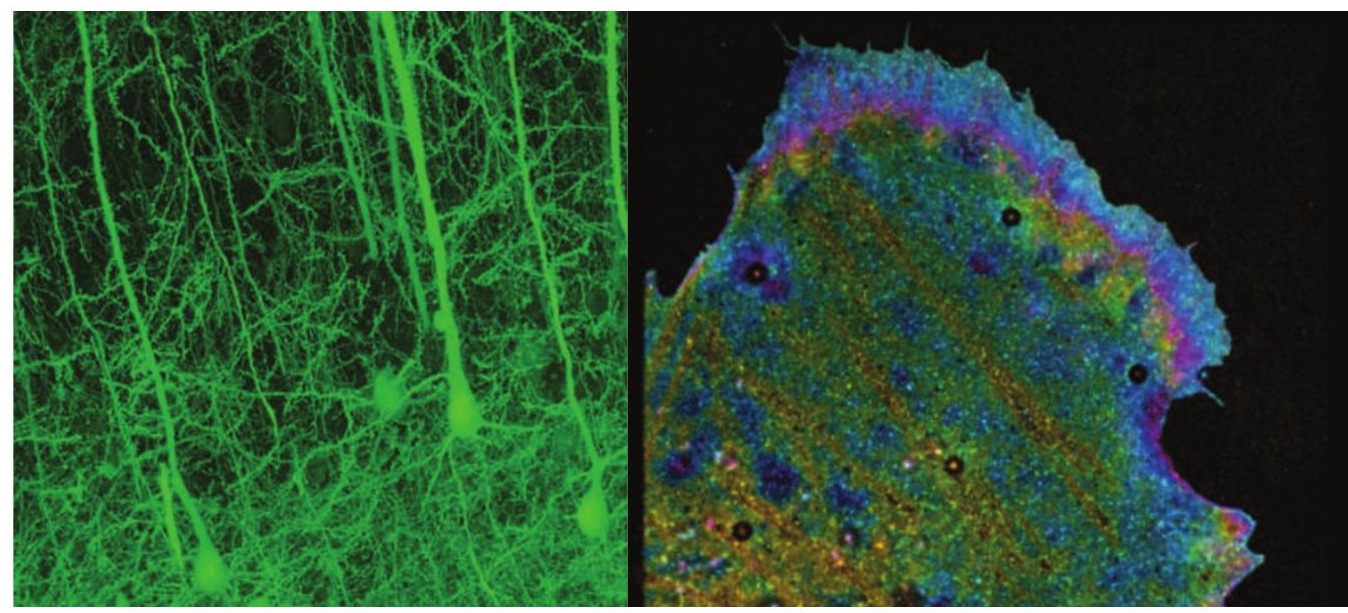

New microscopy techniques are showing structures (pyramidal neurons, left and a U2OS bone cell, right) in unprecedented detail.

mammalian cell nuclei tagged with fluorescent nanoparticles, obtaining a resolution of less than $70 \mathrm{~nm}$ in the focal plane ${ }^{1}$.

Bernardo Sabatini, a neurobiologist at Harvard Medical School in Boston, Massachusetts, used the same combination to image slender neuronal projections and dendritic spines in brain slices, which are not visible using conventional methods ${ }^{2}$. Sabatini and his team built the system themselves. They created the ring shape in the depletion laser using a phase plate to split the laser light so that one part served as the central spot and the other part as the ring. The result was a three-fold improvement in spatial resolution over normal two-photon techniques.
Advances in resolution have not been

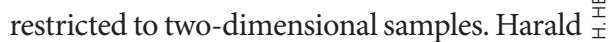
Hess and his colleagues at Howard Hughes Medical Institute's Janelia Farm Research Campus in Virginia have modified PALM to do 3D imaging by adding an interferometer and a second objective lens ${ }^{3}$. Called iPALM, this method can pinpoint fluorescent labels to within 10-20 nm in three dimensions. However, the samples must be around 225-250 $\mathrm{nm}$ thick, and ensuring that the extra optics needed are perfecty aligned is a time-consuming task. As a result, iPALM might prove challenging to commercialize, Hess says.

Groups led by Moerner and Rafael Piestun at the University of Colorado, Boulder, have

\section{BREAKING THE LIGHT BARRIER}

In 1873, German physicist Ernst Abbe proposed that diffraction fundamentally limited the resolution that any microscope could achieve to around half the wavelength of light. And, despite many advances, microscopes didn't threaten to challenge this law of physics for more than a century. Stefan Hell, now a director of the Max Planck Institute for Biophysical Chemistry in Göttingen, Germany, was the first to show that the diffraction limit could be beaten.

Hell, while a postdoc at the University of Turku in Finland in the 1990s, thought that, with the right lasers, he could activate a fluorescent spot and then shrink it by superimposing a larger, hollow beam of light to deplete all the light emission except for that at the centre of the spot. He called the technique stimulated emission depletion (STED) microscopy. Although many physicists were initially sceptical of Hell's ideas, by 2000 he had used STED to produce the first nanoscale fluorescence images ${ }^{10}$.

Super-resolution microscopy has blossomed since, allowing researchers to see cellular processes unfolding at nanometre scales. "This is something that the field has desired since people first started looking through light microscopes," says Jan Liphardt, a biophysicist at the Lawrence Berkeley National Laboratory in California.

Since Hell's work, the field has been boosted by other groups, including those of Eric Betzig, a physicist at the Howard Hughes Medical Institute's Janelia Farm Research Campus in Virginia and Jennifer Lippincott-Schwartz, a cell biologist at the National Institutes of Health in Bethesda, Maryland. In 2006, the groups reported that they had increased resolution by harnessing single-molecule photoactivatable fluorescent proteins and compiling images of thousands to millions of them" They called the approach photoactivated localization microscopy (PALM).

At Harvard University in Cambridge, Massachusetts, physicist Xiaowei Zhuang has developed three-dimensional (3D) stochastic optical reconstruction microscopy (STORM), which uses photoswitchable probes to temporally separate the overlapping images of individual molecules and so boost resolution to ten times better than the diffraction limit.

Yet another approach fluorescence PALM (FPALM) was developed in 2006 by Samuel Hess, a physicist at the University of Maine in Orono. His group's technique involves looking at thousands of fluorophores at once, and localizing on small numbers at a time. These methods have already begun to demostrate their utility. For example, in 2007, Hess's group showed that FPALM could be used to detect proteins clustering in lipid rafts in living cells ${ }^{12}$. In 2008, Lippincott-Schwartz's group combined PALM with singleparticle tracking to detect the movement of membrane proteins in live cells ${ }^{13}$. And Zhuang's group used 3D STORM to image microtubules and other molecular structures within monkey kidney cells $^{14}$, later extending the method to multicolour 3D imaging of whole cells ${ }^{15}$. Hell's group, early in 2008 , used the STED method to show the movement of synaptic vesicles inside living neurons at video rate $^{16}$.

But the field is just warming up. "To people like me who were trained in physics or optics in the 1990 s, it's just unbelievable that one can image below the resolution of light," says Bernardo Sabatini, a neurobiologist at Harvard Medical School in Boston, Massachusetts. "The major revolution for the next 5 or 10 years is getting these advances to answer biological questions." 
come up with another 3D technique, called double-helix PALM. This adds two lenses and a spatial light modulator to a standard wide-field microscope. The modulator splits the light into a double-helix shape that, when shined on to a fluorescent tagged molecule, generates two spots of light. The angular orientation of the spots can be used to calculate the depth of the molecule. "The double helix has a much deeper depth of field over which the objects remain in focus," says Moerner. "In current experiments we use a two-micrometre range, but that can be changed depending on how the phase modulator is designed."

In collaboration with Robert Twieg at Kent State University in Ohio, Moerner's team has localized molecules with $20 \mathrm{~nm}$ precision in depth and $12 \mathrm{~nm}$ precision in length and width ${ }^{4}$, and has used the system to image bacterial proteins whose structures have never before been seen in cells.

\section{Going live}

SIM is also finding application in 3D systems - through imaging live cells. Mats Gustafsson and his colleagues at Janelia Farm sped up the bar-code patterns used in two-dimensional SIM to allow it to image live cells, which are constantly moving. They did this with a liquidcrystal spatial light modulator, a mirror-like device, 1.5 centimetres long, that generates patterns of light using thousands of pixels that can be controlled individually. The modulator allows the microscope to generate new patterns of light about 1,000 times faster than the normal SIM equipment. The technique has allowed Gustafsson's team to see proteins moving along individual microtubules within living cells of the fruitfly Drosophila melanogaster at $100 \mathrm{~nm}$ resolution ${ }^{5}$.

SIM will soon become even faster because of advances in camera technology. "You can already buy cameras that go up to 1,000 frames per second, and that speed can more or less be directly translated into speed increase in structured illumination," says Rainer Heintzmann, a molecular biologist at King's College London, who has used the system to see the inner folds of a cell's mitochondria.

One of the main hurdles to widespread use of superresolution microscopy in biology is the lack of appropriate probe molecules. Developing new probes is a trial-and-error process of seeing whether each protein variant will work in a variety of biological samples. "This is where the long, hard

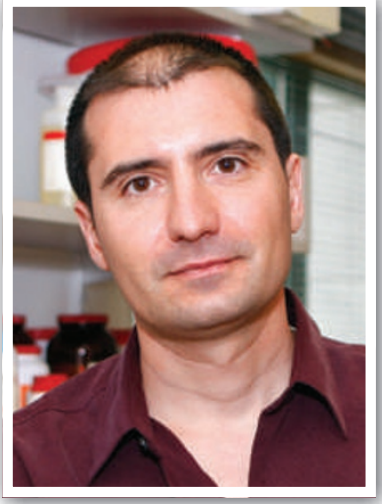

Bernardo Sabatini has modified microscopy software.
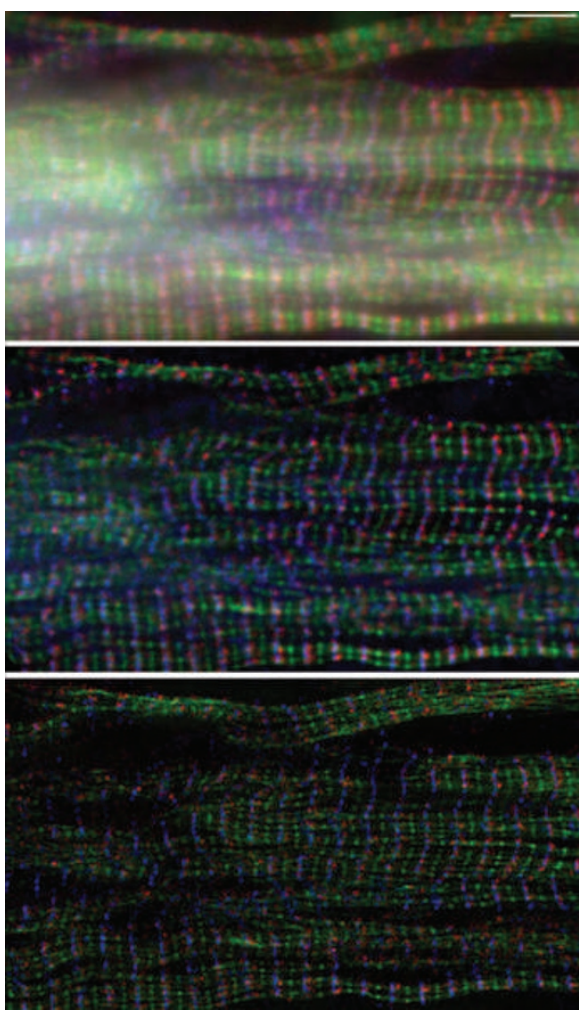

Increasing detail: skeletal muscle cells seen by wide-field fluorescence image (top); standard deconvolution image; and by Applied Precision's DeltaVision OMX system (bottom).

to track more than one protein in a cell. Twocolour experiments can work but, at present, the second colour has usually faded by the time the first colour has been completed, Hess says.

A group led by Vladislav Verkhusha, an associate professor of anatomy and structural biology at Albert Einstein College of Medicine at Yeshiva University in New York, has developed a new red probe ${ }^{6}$ that works using PALM methods. Called photoactivatable monomeric cherry, or PAmCherryl, the protein becomes red when activated by violet light. It seems to have better $\mathrm{pH}$ stability, faster photoactivation and better photostability than other red photoactivatable probes, the group says.

Several groups are working on small organic probes that can be used with PALM or STORM. Earlier this year, for example, researchers at Bielefeld University in Germany imaged the inner membranes of mitochondria ${ }^{7}$ using commercially available fluorophores. The researchers triggered the fluorophores to light up on work is," Hess says. "Right now there are few examples of single colours, but making them work in pairs will be the part that will bring a lot of value to the field." Having two or more colours would help biologists by allowing them cue by altering the chemical environment.

And Hell is backing an unlikely material as a potential probe: diamond. His team found that STED microscopy can pick up subtle structural defects in diamonds with 10-20 nm resolution ${ }^{8}$. So if the diamonds can be ground into 35-nanometre-sized pieces and attached to antibodies - which researchers have shown is possible - they could be used as tags that will never bleach under STED microscopy's lasers. There is a "clear prospect" of using diamonds for cell-biology applications, Hell says. "It is not a pipe dream. It's farther down the road but is coming."

\section{Applying software}

An increasingly important aspect of microscopy - particularly super-resolution systems - is the software that constructs the final images. Scientists who want to modify the output or improve the accessibility of their superresolution systems can develop algorithms and write programs for image processing. Many scientists have developed their own software and made it available to the community. "The design of the microscope and its modification is available to anybody who knows how to program," says Sabatini, and once you know how the microscope works, it is possible to determine where the photons should be and, from that, how the image can be constructed.

Sabatini has helped at least ten other labs to implement super-resolution methods. "Biologists feel comfortable taking the software we wrote and modifying it for their application," he says. "In our own lab, a lot of the advances we have week to week are software driven. We get an idea for an experiment, and we just reprogram the code." That makes experiments much less expensive, and the weekly progress wouldn't be possible using off-the-shelf systems, he adds.

Zhuang's group has created software that allows image processing to happen as the data are acquired. Unlike a conventional fluorescence image, a single STORM image is obtained by stacking many frames. "It used to be that we could only do post-image processing," she says. "Now we have analysis software that you can use to see the build-up of the high-resolution image as it is being recorded." This will speed up experiments, she says, because if there is something about the sample you dislike, you can change it before the image construction is finished.

\section{One-stop system?}

Biologists still have a long wish list for superresolution imaging technology, probes and software. "We're at least one or two years away from the technology being fully established," says Jan Liphardt, a biophysicist at the University of California, Berkeley. "My own preference is to keep building my own hardware."

Although improvements are happening fast, and developers are quickly adding to the pool of $3 \mathrm{D}$ and live-cell imaging data, there is still no one system that can readily do everything a biologist wants, says M. Cristina Cardoso, a cell biologist at the Technical University of Darmstadt, Germany.

Cardoso and her colleagues used 3D-SIM 
and spatially modulated illumination to compare the sizes of DNA replication spots called replicons - on chromosomes within a cell nucleus, and how they change over time. Replicons are, on average, $125 \mathrm{~nm}$ in diameter. Although these super-resolution techniques allowed her to identify three- to fivefold more replicons than the numbers previously reported ${ }^{9}$, the researchers would have preferred to conduct the experiment in live cells to get better temporal resolution. However, with current systems this would have meant giving up some spatial resolution, so instead they stopped replication and fixed the cells at various points in their cycle.

\section{Rivalling Hubble}

Live-cell approaches are improving, but there are still challenges. In STORM, PALM and SIM, multiple pictures must be taken and then blended together to reveal all of the detail. "Does that represent structure or motion of some sort?" Moerner says, adding that it is an ongoing challenge to distinguish the two.

The challenges have not stopped large collaborations from harnessing super-resolution microscopes to answer big questions. Liphardt says that the University of California, Berkeley, has seven to ten PALM/STORM systems, built by researchers there and at other labs, that will be used for research into tumour-cell signalling as part of a US\$15.7-million grant awarded by the US National Cancer Institute.

About a year ago, a consortium formed, which includes Zhuang and Smith, to construct a detailed map of neuronal connections in the brain. This 'connectome' project will combine super-resolution microscopy, including STORM, with array tomography and electron microscopy to see neuronal details at a resolution of $100 \mathrm{~nm}$ or better over the entire brain of a mouse, and eventually a human. To get a complete wiring diagram of the brain, says Zhuang, one needs to resolve the long neuronal projections - axons - that are invisible under a traditional light microscope.

"We're starting to get pictures out of the mouse brain that rival anything from the Hubble Space Telescope, and we're just getting started," Smith says. But to make use of that

Conventional
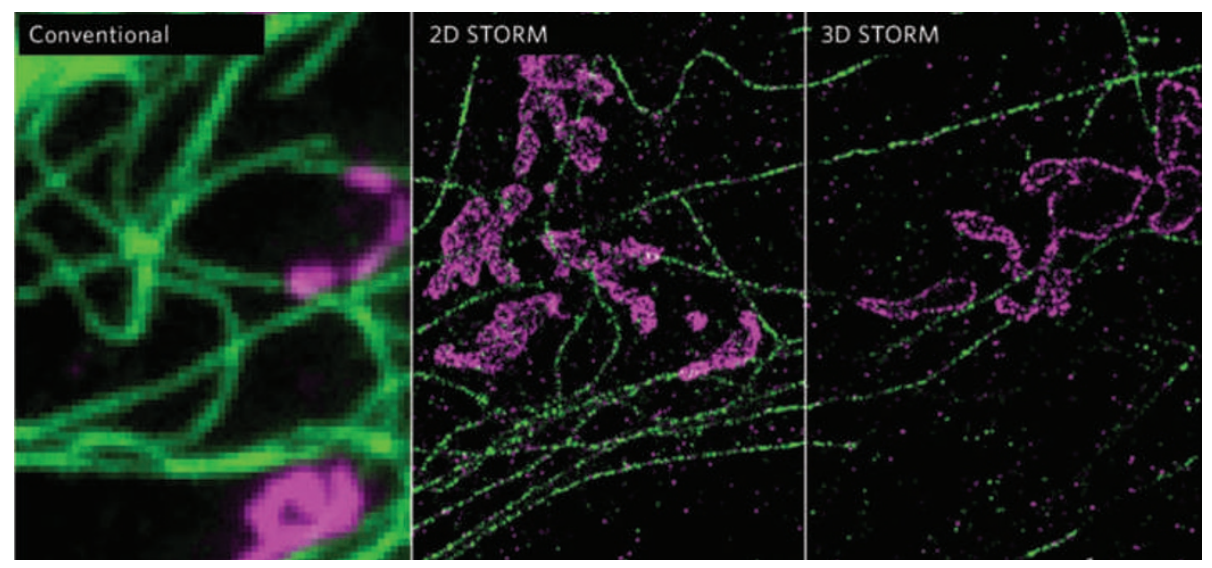

Composite two-colour image of microtubules (green) and mitochondria (magenta) in a cell.
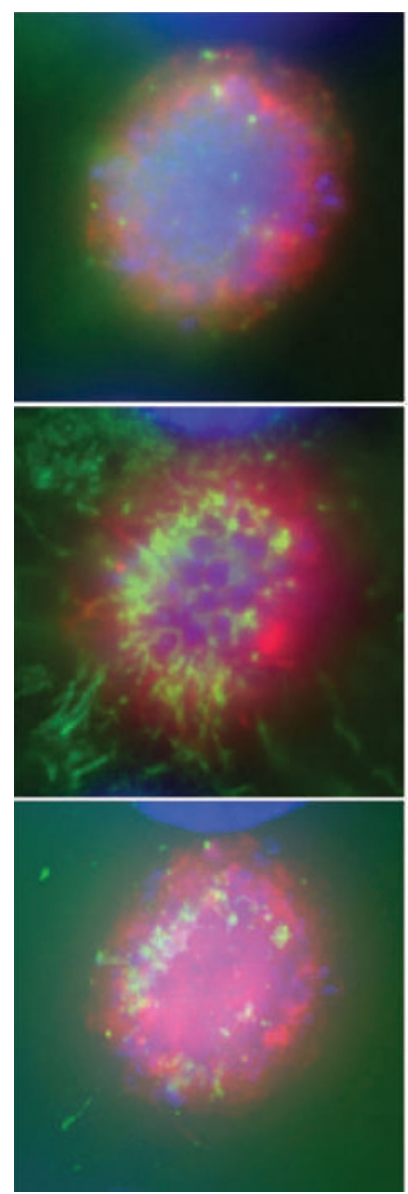

information will require a lot more than looking at pretty pictures: the new imaging data will also push forwards development of software used to reconstruct and analyse connections in three dimensions. "The tools for comparing and understanding the elaborate circuit diagrams are beyond anything that anyone has thought of today," he says.

The promise of super-resolution microscopy - thought for so long to be little more than a dream - is starting to become a reality. Researchers have taken different approaches and are using tools and techniques borrowed from physics, chemistry and computing
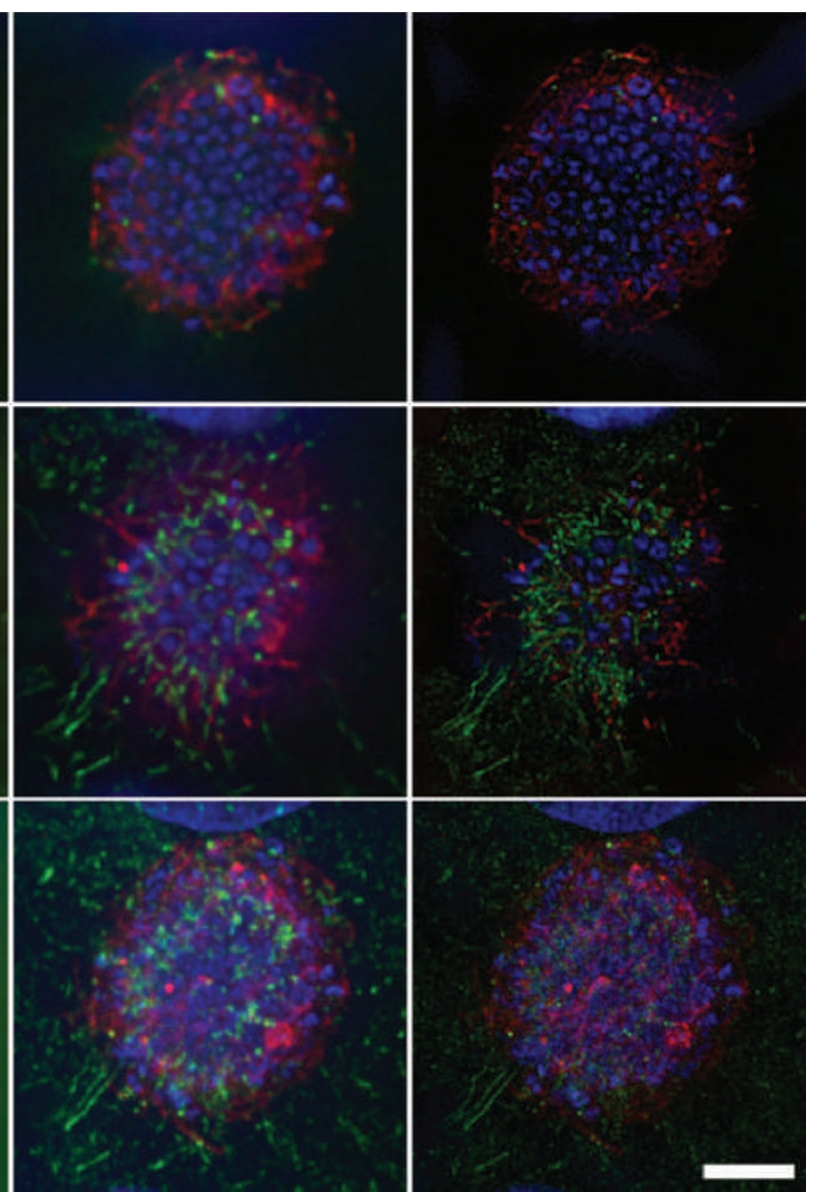

Epithelial cells seen by (left to right) wide field, deconvolution and OMX microscopy. Scale bar: $4 \mu \mathrm{m}$. Top row, midplane through microcolony; centre, bottom of colony; bottom, maximum intensity projections.

technology to bring the nanoscopic world to our macroscopic eyes. Although commercialization is progressing, there is still plenty of room for the do-it-yourself biologist to modify and improve their systems, and produce images of stunning complexity that will rival anything else in science.

Kelly Rae Chi is a freelance journalist based in Cary, North Carolina, USA.

1. Moneron, G. \& Hell, S. W. Opt. Express 17, 14567-14573 (2009).

2. Ding, J. B., Takasaki, K. T. \& Sabatini, B. L. Neuron 63, 429-437 (2009).

3. Shtengel, G. et al. Proc. Natl Acad. Sci. USA 106, 3125-3130 (2009).

4. Pavani, S. R. et al. Proc. Natl Acad. Sci. USA 106, 2995-2999 (2009).

5. Kner, P., Chhun, B. B., Griffis, E. R., Winoto, L. \& Gustafsson, M. G. Nature Meth. 6, 339-342 (2009).

6. Subach, F. V. et al. Nature Meth. 6, 153-159 (2009).

7. van de Linde, S. et al. Photochem. Photobiol. Sci. 8, 465-469 (2009).

8. Han, K. Y. et al. Nano Lett. 9, 3323-3329 (2009)

9. Baddeley, D. et al. Nucleic Acids Res. doi:10.1093/nar/ gkp901 (2009).

10. Klar, T. A., Jakobs, S., Dyba, M., Egner, A. \& Hell, S. W. Proc. Natl Acad. Sci. USA 97, 8206-8210 (2000)

11. Betzig, E. et al. Science 313, 1642-1645 (2006)

12. Hess, S. T. et al. Proc. Natl Acad. Sci. USA 104, 17370-17375 (2007).

13. Manley, S. et al. Nature Meth. 5, 155-157 (2008).

14. Huang, B., Wang, W., Bates, M. \& Zhuang, X. Science 319, 810-813 (2008).

15. Huang, B., Jones, S. A., Brandenburg, B. \& Zhuang, X. Nature Meth. 5, 1047-1052 (2008)

16. Westphal, V. et al. Science 320, 246-249 (2008) 